\title{
Twelve-month follow-up of left atrial appendage occlusion with Amplatzer Amulet
}

\author{
Caroline Kleinecke, Jai-Wun Park, Martin Gödde, Konstantin Zintl, \\ Steffen Schnupp, Johannes Brachmann
}

Klinikum Coburg, Medical Clinic II (Cardiology/Intensive Care Medicine), Coburg, Germany

\begin{abstract}
Background: The Amplatzer Amulet (St. Jude Medical, Minneapolis, MN, USA) is a second generation Amplatzer device for left atrial appendage (LAA) occlusion (LAAO) for stroke prophylaxis in patients with atrial fibrillation. This research sought to assess the clinical performance of the Amplatzer Amulet device and in follow up for 12 months.

Methods: In this single-center registry patients with atrial fibrillation and contraindication to oral anticoagulation underwent LAAO with the Amplatzer Amulet device. Follow-up was performed before discharge, by transesophageal echocardiography (TEE) after 6 weeks and telephone interview after 3, 6 and 12 months.

Results: Between October 2014 and August 201550 patients (76.1 \pm 8.3 years; 30 male) were enrolled. Procedural success was achieved in $49(98 \%)$ patients. Major periprocedural adverse events were observed in 4 (8\%) of patients: 1 device embolization, 2 pericardial effusions requiring pericardiocentesis and 1 prolonged hospital stay due to retropharyngeal hematoma from the TEE probe. Follow-up TEE was available in 38 of 50 patients showing complete LAA sealing in all. 2 device-related thrombi were also documented. At 12-month follow-up 7 patients had died unrelated to the device. Ischemic stroke occurred in 3 patients. According to neurological examination two were classified as microangiopathic and not cardio-embolic. The other one could not be classified. Bleeding complications (5 minor, 3 major) were documented in 8 patients.

Conclusions: Although minimizing procedure-related complications remains challenging, LAAO with the Amplatzer Amulet device showed high procedural success and excellent LAA sealing. (Cardiol J 2017; 24, 2: 131-138)
\end{abstract}

Key words: left atrial appendage occlusion, Amplatzer Amulet, atrial fibrillation, stroke prophylaxis

\section{Introduction}

Thrombus in the left atrial appendage (LAA) is the principle cause of cardio-embolic events such as stroke in patients with non-valvular atrial fibrillation (AF) [1]. The randomized Protect AF and PREVAIL trials showed that LAA occlusion (LAAO) with the Watchman device (Atritech, Boston Scientific, Natick, MA, USA) is an alternative for oral anticoagulation (OAC) for stroke prophylaxis in patients with $\mathrm{AF}[2,3]$.
The first generation Amplatzer Cardiac Plug (ACP, St. Jude Medical, Minneapolis, MN, USA) was first introduced in 2008. It consists of two major components: a distal lobe that secures the device in the LAA body and a proximal disc that covers the ostium [4]. A multicentre registry of 1,047 patients demonstrated high procedural success $(97.3 \%)$ and a low annual rate of systemic thrombembolism (2.3\%) [5].

The Amplatzer Amulet (St. Jude Medical, Minneapolis, MN, USA) is the second generation

Address for correspondence: Dr. Caroline Kleinecke, Klinikum Coburg, Medical Clinic II (Cardiology/Intensive Care Medicine) Ketschendorfer Straße 33, 96450 Coburg, Germany, tel: +49 956122 33215, fax: +49 9561226419 , e-mail: carolinekleinecke@web.de

Received: 25.11.2016 Accepted: 15.01.2017 


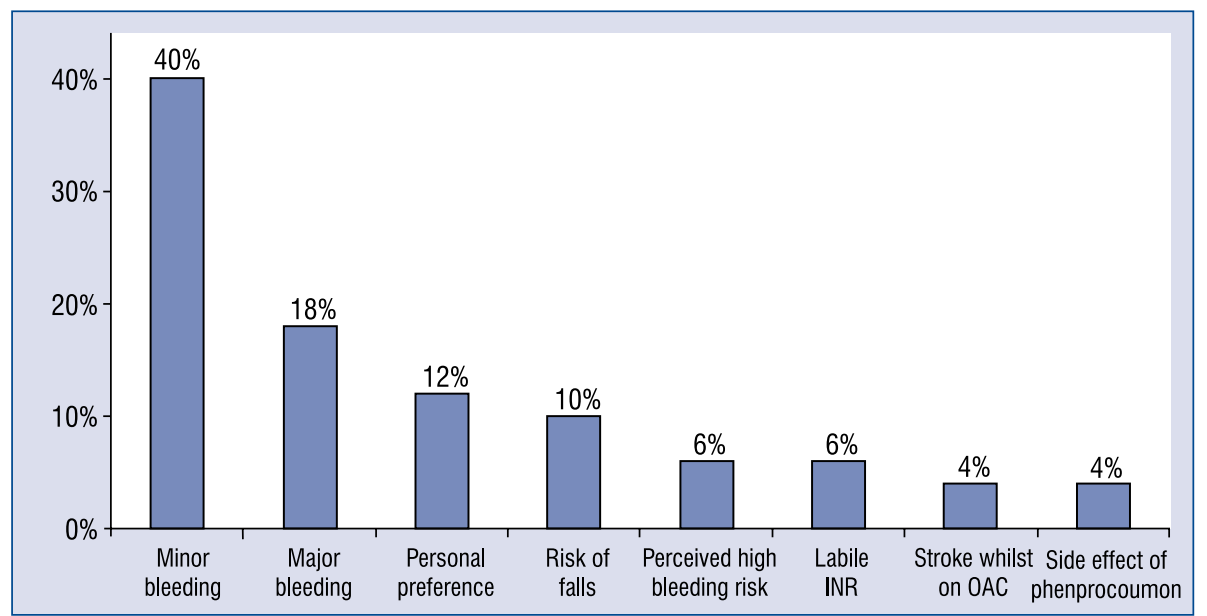

Figure 1. Indications for left atrial appendage occlusion; INR — international normalized ratio; OAC — oral anticoagulation.

Amplatzer device that has been developed to facilitate implantation and to reduce device-related complications like embolisation or leak [6].

First series of LAAO with the Amplatzer Amulet showed similar procedural and short-term clinical outcomes compared with the ACP [7-10]. In the series of Abualsaud et al. [11] with $50 \mathrm{pa-}$ tients the Amulet was associated with a significant reduction in residual leaks at follow-up.

In this single-centre registry it was sought to assess the clinical performance of the Amplatzer Amulet device at 12-months follow-up.

\section{Methods}

All consecutive patients who underwent LAAO with the Amplatzer Amulet at the Department of Cardiology, Klinikum Coburg, Germany between October 2014 and August 2015 were retrospectively enrolled in the study. Patient characteristics, procedural and echocardiographic data as well as adverse events were collected in a database. Eligibility criteria for LAAO were AF (paroxysmal, persistent or permanent) with indication for OAC $\left(\mathrm{CHA}_{2} \mathrm{DS}_{2}\right.$-VASc Score $\left.\geq 1\right)$ and contraindication for $\mathrm{OAC}$ or the patient's explicit request for LAAO as alternative to OAC. Exclusion criteria were thrombus in the LAA, planned cardiac surgery, previous closure of the interatrial septum by surgery or device implantation, active endocarditis or other serious infections. All patients provided written informed consent before the procedure. This registry was initiated by the investigators. There was no external funding.

\section{Amplatzer Amulet}

The Amplatzer Amulet has undergone several changes in relation to the ACP. The self-expanding device is made of braided nitinol mesh. It consists of a lobe and a disc connected by a central waist. The distal lobe anchors through stabilizing wires within the so called landing zone of the LAA. The proximal disc covers the LAA orifice (Fig. 1).

Eight sizes (smaller devices: 16, 18, 20, $22 \mathrm{~mm}$, larger devices: 25, 28, 31, $34 \mathrm{~mm}$ ) accommodate varying LAA anatomies with landing zone diameters from $13 \mathrm{~mm}$ to $31 \mathrm{~mm}$. An increased lobe length (smaller devices: $7.5 \mathrm{~mm}$, larger devices: $10 \mathrm{~mm}$ ) and a higher number of stabilizing wires in larger devices increase the overall stability.

The disc diameter (smaller devices: lobe + $+6 \mathrm{~mm}$, larger devices: lobe $+7 \mathrm{~mm}$ ) has been increased for better coverage of the LAA orifice. The end screw on the disc has been recessed to avoid thrombus formation in the LAA. The waist length (smaller devices: $5.5 \mathrm{~mm}$, larger devices: $8 \mathrm{~mm}$ ) between lobe and disc has been increased for more flexible placement of the lobe within the LAA.

The device is pre-loaded in a loader and preattached to the delivery cable. This setup facilitates loading into the delivery sheath and is intended to minimize air embolism. The device is delivered to the LAA through a TorqVue $45^{\circ} \times 45^{\circ}$ double-bend sheath (sizes: $16-25 \mathrm{~mm}$ : 12 or 14 French with adapter, sizes 25-34 mm: 14 French) [12].

\section{Procedure}

Transesophageal echocardiography (TEE) (Vivid E9 and Vivid S6, GE Healthcare, Milwaukee, 
USA) was performed before and during the procedure to rule out LAA thrombus and to determine LAA size with measurement of the orifice, landing zone and depth from different angles $\left(0^{\circ}, 45^{\circ}, 90^{\circ}\right.$, $130^{\circ}$ and $\left.180^{\circ}\right)$. One operator was expert $(>1000$ procedures), 1 operator was experienced ( $>50$ procedures) and 1 operator was new to the procedure ( $<5$ procedures). All procedures were performed with local anesthesia and under conscious sedation. After venous access and TEE guided transseptal puncture heparin was administered (activated clotting time $>250 \mathrm{~s}$ ). All procedures were performed via transseptal puncture in inferoposterior location to achieve perpendicular sheath angulation with respect to the long axis of the LAA. Since a patent foramen ovale (PFO) is usually located at a more anterior and superior position, in case of a PFO, care was taken not to puncture through the PFO. LAA angiogram was performed with a pigtail catheter in right anterior oblique caudal and cranial projections. For choosing the appropriate Amulet device both angiographic and echocardiographic measurements were taken into account oversizing the device from $3 \mathrm{~mm}$ to $5 \mathrm{~mm}$ with respect to the largest measured landing zone. The device was advanced on the delivery cable through the delivery sheath and positioned into the LAA. Tug test was performed under fluoroscopy by pulling and pushing the delivery cable. To intensify this security test in challenging LAA anatomies (shallow LAA with big orifice) the continuous ( $>10 \mathrm{~s}$ ) tug test was performed. During this maneuver the delivery sheath is retracted to a stable, but relaxed position within the LA without any rotation tension until the tip of the sheath is significantly separated from the LAA orifice. Then the delivery cable is gently, steadily, and continuously pulled back until the disc of the Amulet device is separated from the LAA orifice and forms the shape of a rugby-ball. Because the stabilizing wires of the Amulet device are located at the distal end of the Amulet lobe, a slight separation between the distal and proximal cage of the Amulet lobe during the continuous tug indicates that the stabilizing wires are trapped in the LAA trabeculi.

Echocardiographic and angiographic criteria for correct deployment were compression of the lobe, a perpendicular axis of lobe and LAA neck, the lobe positioned two thirds distal to the circumflex artery, separation between lobe and disc, a concave shape of the disc. Coverage of the orifice and residual leaks were evaluated by echocardiography and injecting contrast media through the delivery sheath. Furthermore, echocardiography evalu- ated impingement of the mitral valve or left upper pulmonary vein and the presence of pericardial effusion. If necessary, the device was repositioned or a different sized device was selected. At last the Amulet device was released.

\section{Follow-up}

After the procedure patients received dual antiplatelet therapy with clopidogrel for 3 months and aspirin for at least 6 months.

Follow-up was performed in hospital by clinical examination and transthoracic echocardiography to rule out device embolisation and pericardial effusion.

Transesophageal echocardiography was performed after 6 weeks to control position and endothelialization of the device, detect residual leaks, and to observe thrombus formation or infectious endocarditis.

Follow-up was done by review of all subsequent clinical records and structured telephone interviews after 3, 6 and 12 months.

\section{Definitions}

Procedural success was defined as successful implantation of the Amplatzer Amulet.

Periprocedural device- or procedure-related major adverse events included immediate procedural mortality ( $<72 \mathrm{~h}$ after the index procedure), stroke/transient ischemic attack (TIA), device embolization, clinically relevant cardiac tamponade or pericardial effusion, fatal or major bleeding, myocardial infarction, systemic embolism, pericarditis and other relevant complications leading to prolonged hospital stay.

Adverse events at follow-up included cardiovascular and non-cardiovascular mortality, stroke/ /TIA and bleeding.

According to the definition of the Bleeding Academic Research Consortium (BARC), [13] bleeding was defined as fatal (type 5) or major with hemoglobin drop of $>3 \mathrm{~g} / \mathrm{dL}$, requirement of packed red blood cell transfusions or intracranial hemorrhage (type 3a-c). Minor bleeding was defined as not actionable (type 1 ) or actionable (type 2) requiring medical intervention, leading to prompt evaluation or hospitalization but not meeting the criteria of major bleeding.

Complete LAA sealing was defined as absence of flow or absence of residual peridevice flow $>5 \mathrm{~mm}$ assessed by color Doppler.

\section{Statistical analysis}

Categorical variables are expressed as frequencies and percentages. Continuous data are 
Table 1. Baseline characteristics $(n=50)$.

\begin{tabular}{lc}
\hline Age [years] & $76.1 \pm 8$ \\
Age $\geq 75$ years & $31(62 \%)$ \\
Male & $30(60 \%)$ \\
Permanent atrial fibrillation & $26(52 \%)$ \\
$\mathrm{CHA}_{2} \mathrm{DS}_{2}$-VASc score & $5.2 \pm 1.8$ \\
HAS-BLED score & $3.5 \pm 0.9$ \\
Previous stroke/TIA & $17(34 \%)$ \\
Peripheral embolization & $4(8 \%)$ \\
Congestive heart failure & $8(16 \%)$ \\
Arterial hypertension & $50(100 \%)$ \\
Diabetes mellitus & $22(44 \%)$ \\
Creatinine [mmol/L] & $62 \pm 22$ \\
Carotid disease & $12(24 \%)$ \\
Coronary artery disease & $35(70 \%)$ \\
\hline
\end{tabular}

Categorical variables are expressed as frequencies $(n)$ and percentages $(\%)$. Continuous data are reported as means and ( \pm ) standard deviation; TIA — transient ischemic attack

Table 2. Procedural data $(n=50)$.

\begin{tabular}{lc}
\hline Procedural success & $49(98 \%)$ \\
Size of Amplatzer Amulet: & $1(2 \%)$ \\
$16 \mathrm{~mm}$ & $1(2 \%)$ \\
$20 \mathrm{~mm}$ & $11(22 \%)$ \\
$22 \mathrm{~mm}$ & $20(40 \%)$ \\
$25 \mathrm{~mm}$ & $11(22 \%)$ \\
$28 \mathrm{~mm}$ & $2(4 \%)$ \\
$31 \mathrm{~mm}$ & $4(8 \%)$ \\
$34 \mathrm{~mm}$ & \\
LAA measurements: & $22 \pm 5$ \\
Opening [mm] & $21 \pm 4$ \\
Landing zone [mm] & $25 \pm 7$ \\
Depth [mm] & \\
LAA morphology: & $24(48 \%)$ \\
Chicken wing & $6(12 \%)$ \\
Windsock & $11(22 \%)$ \\
Cauliflower & $4(8 \%)$ \\
Cactus & $5(10 \%)$ \\
Cone & $13 \pm 7$ \\
Implantation attempts: & $38(76 \%)$ \\
2 & $11(22 \%)$ \\
Fluoroscopy time [min] & $1(2 \%)$ \\
Contrast media [mL] & $13 \pm 56$ \\
\hline
\end{tabular}

Categorical variables are expressed as frequencies $(n)$ and percentages $(\%)$. Continuous data are reported as means and $( \pm)$ standard deviation; LAA — left atrial appendage reported as means and standard deviation. Descriptive statistical analysis was performed with SPSS 17.0 (SPSS Inc., Chicago, Illinois, USA).

\section{Results}

\section{Baseline characteristics}

Between 01/10/2014 and 24/08/2015 a total of 50 patients $(76.1 \pm 8.3$ years; 30 male) were enrolled. Twenty six (52\%) patients had permanent AF. The mean $\mathrm{CHA}_{2} \mathrm{DS}_{2}$-VASc score was $5.2 \pm 1.8$ and the mean HAS-BLED score was $3.5 \pm 0.9$.

Baseline patient characteristics are summarized in Table 1. Indications for LAAO are shown in Figure 1.

\section{Procedural data}

Procedural data are summarized in Table 2. Procedural success was achieved in 49/50 (98\%) patients. In 1 patient device embolization occurred on the day of the procedure.

\section{Periprocedural major adverse events}

Periprocedural major adverse events were observed in 4 (8\%) patients. One device embolization occurred on the day of the procedure and required cardiac surgery. The procedure had been performed by the least experienced operator $(<5$ interventions). Retrospective analysis of fluoroscopy revealed peridevice leak post-implantation (Fig. 2, 1A-C). At 6-month follow-up the patient reported full recovery.

One cardiac tamponade was drained immediately. One pericardial effusion required pericardiocentesis at day 22 after the procedure. Whether cardiac tamponade or pericardial effusion were procedure or device-related is unknown.

One patient experienced prolonged hospital stay (33 days) due to retropharyngeal hematoma from the TEE probe necessitating mechanical ventilation.

Five access site complications $(10 \%)$ included 1 bleeding type 2 and 1 bleeding type 3a according to the BARC bleeding definition, 2 femoral artery pseudoaneurysms and 1 combined pseudoaneurysm and bleeding type 3a. All pseudoaneurysms were treated with thrombin injection. Acute renal injury stage 1 in 1 patient was treated with intravenous fluids and led to prolonged hospital stay (6 days).

\section{TEE follow-up}

Follow-up TEE, routinely performed after 6 weeks, was available in 38 out of 50 patients 


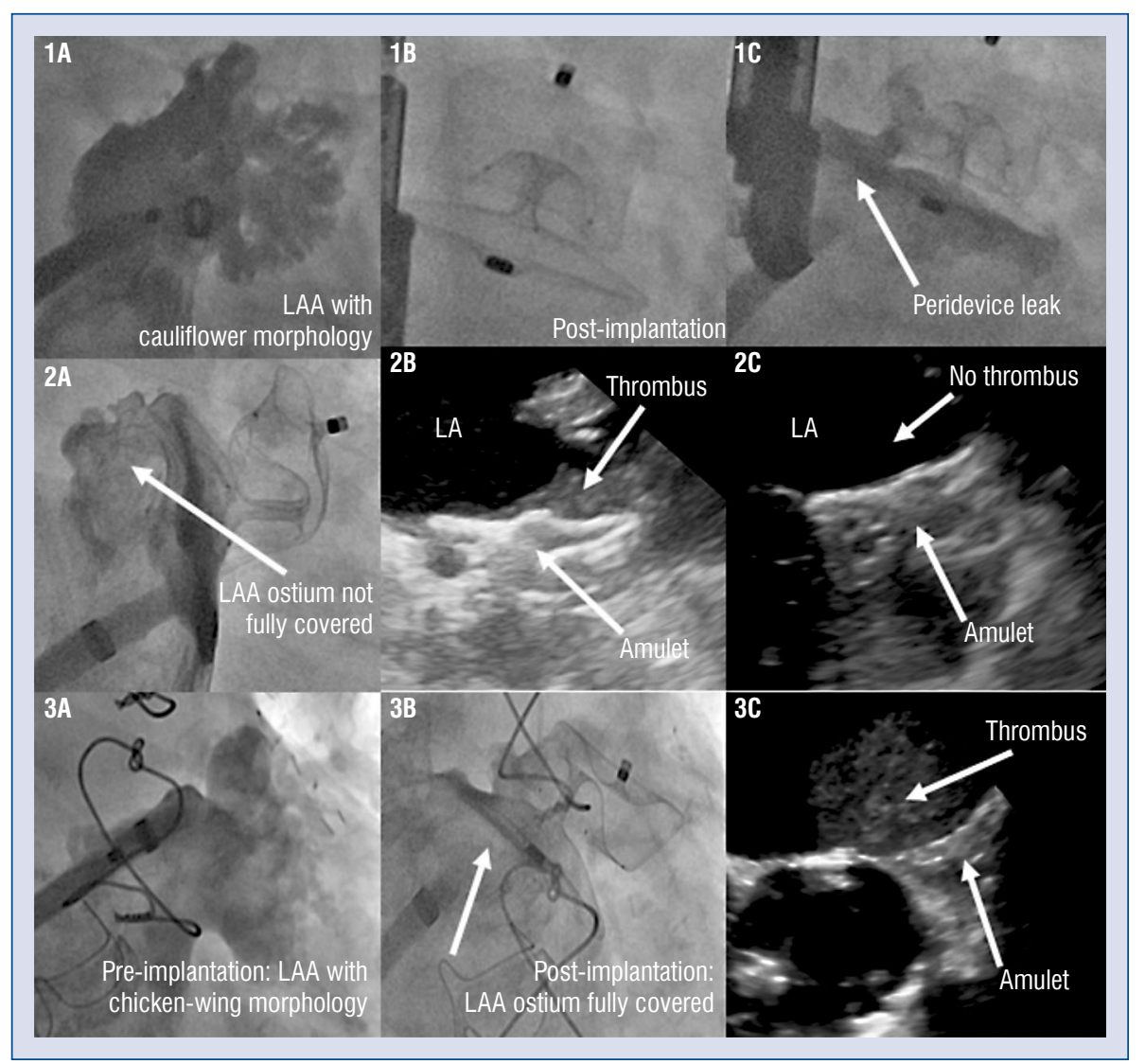

Figure 2. Details of patients with device embolization and thrombus formation; 1A-C. Patient with device embolization showing left atrial appendage (LAA) cauliflower morphology pre-implantation (1A). Deployment criteria of lobe and disc seem fullfilled post-implantation (1B), but angiogram reveals peridevice leak (1C); 2A-C. Patient with device thrombosis. X-ray post-implantation shows a remaining pouch of the LAA ostium which was not fully covered (2A). Transesophageal echocardiography after 6 weeks reveals a thrombus on the device (2B). After another 6 weeks of oral anticoagulation the thrombus has dissolved (3A); 3A-C. Patient with device thrombosis showing LAA chicken-wing morphology pre-implantation (3A). X-ray post-implantation shows full coverage of the LAA ostium (3B). During 12 months follow-up a large thrombus was detected on the device (3C); LA — left atrium.

(76\%). Two patients had died before TEE follow-up. Several patients refused TEE follow-up because of comorbidities and frailty. TEE showed complete LAA sealing in all available studies.

In 1 patient device-related thrombus was found in routinely performed TEE after 6 weeks. It disappeared after 6 weeks of treatment with OAC. Retrospective analysis of fluoroscopy showed a cauliflower morphology of the LAA. To avoid embolization the device had been implanted relatively deeply with the disc positioned partially in the neck of the LAA with the ostium of the LAA not fully covered. Thrombus developed in the remaining pouch between the LAA ostium and the disc (Fig. 2, 2A-C).

The second patient was 79 years old who in the first instance had refused TEE control after 6 weeks. In a later hospital stay during 12 -months follow-up TEE detected a relatively large thrombus of $17 \times 8 \mathrm{~mm}$. Implant result on angiogram showed correct deployment criteria of the device with full coverage of the LAA ostium (Fig. 2, 3A-C). Therefore, the exact cause of thrombus formation remains unknown. However, non-adherence to dual antiplatelet therapy seemed possible due to moderate dementia (mini mental test 16 points).

Cardiac surgery was discussed by the local heart team. Due to frailty and comorbidities lifelong treatment with OAC was considered the preferred approach. At 12-months follow-up there were no neurological complications, the patient refused control TEE.

\section{2-month follow-up}

At 12-month follow-up 7 (14\%, 14 cases per 100 patient-years) patients $(83.9 \pm 4.1$ years $)$ had died unrelated to the device or the procedure. 
Patients aged 90, 82, 86 and 85 died as a result of sepsis due to urinary tract infection (2 patients), spontaneous bacterial peritonitis and soft tissue infection 2, 8, 12 months ( 2 patients) after the index procedure.

Other causes of death in 90,79 and 82 year old patients were atrioventricular-block degree III, decompensated chronic heart failure and acute on chronic kidney failure on day 11, 8 and 6 months after device implantation.

An autopsy was not performed in any of these patients nor was a TEE to rule out endocarditis.

However, TTE, which was performed in all patients, showed a regular position of the Amulet device, and there was no suspicion of endocarditis.

Ischemic stroke occurred in 3 (6.1\%, 6 cases per 100 patient-years) patients. TEE in all 3 patients showed complete sealing of the LAA and device-related thrombus was ruled out. National Institutes of Health Stroke Scale (NIHSS) score was evaluated by an independent neurologist. An 83-year-old patient suffered from a disabling, microangiopathic stroke (NIHSS score 7). A nondisabling, microangiopathic stroke (NIHSS score 4) occurred in a 76-year-old patient. A non-disabling stroke with paraphasia (NIHSS score 2) was documented in a 75-year-old patient. Cranial computed tomography scan showed a small lesion in the caput nuclei caudati. It could not be classified as microangiopathic or cardio-embolic. All 3 patients were treated with aspirin.

Eight bleeding complications $(16 \%, 16$ cases per 100 patient-years) were reported. One minor and 2 major bleedings occurred under dual antiplatelet therapy. Major bleeding complications included 1 case of gastrointestinal bleeding and 1 case of subarachnoid bleeding without neurological deficit after a fall. One minor gastrointestinal bleeding and 1 minor hemoptysis occurred under therapy with aspirin alone. Two minor and 1 major gastrointestinal bleedings occurred without antithrombotic therapy.

\section{Discussion}

This study is the first to report 12 -month follow-ups of the Amplatzer Amulet. It showed high procedural success (98\%) and excellent LAA sealing rates $(100 \%)$. There was a relevant rate of major periprocedural complications (8\%). At 12-months follow-up no device-related complications were observed.

Procedural success in our registry was similar to other series published using the Amplatzer
Amulet. These series with 17 [7], 20 [8], 24 [9] and 50 [10] patients reported 100\%, 100\%, 96\% and $94 \%$ procedural process. The Amulet observational study with 1,073 patients reported $98.8 \%$ implant success [14]. Since the multicentre-study of 1,047 patients using the first generation ACP [5] documented $97.3 \%$ successful implantations, these data suggest that procedural success of Amplatzer Amulet and ACP is comparable.

Herein is documented a relevant rate of major periprocedural complications including 1 device embolization, 2 pericardial effusions requiring pericardiocentesis and 1 prolonged hospital stay due to retropharyngeal hematoma from the TEE probe. In this study device embolization occurred in a procedure performed by an operator who was new to the procedure ( $<5$ interventions). Retrospective analysis of fluoroscopy showed a relevant peridevice leak after deployment of the device (Fig. 2, 1A-C). Prolonged hospital stay due to retropharyngeal hematoma from the TEE probe was considered as a serious adverse event. Research indicated that other registries documented only major adverse events which were strictly related to the device and the LAAO procedure itself.

This data reflects a real-world scenario in a challenging patient population (mean age $76 \pm$ \pm 8 years). Furthermore, the above described device embolization and retropharyngeal hematoma caused by the TEE probe reflect the learning curve at this center. To avoid retropharyngeal hematoma, this center in recent times administers heparin after introducing the TEE probe and, respectively, the procedure is even stopped if there are difficulties introducing the TEE probe.

The Protect AF trial and the continued access registry [15] showed a significant improvement of Watchman LAA closure and decreased rate of procedure-related complications with increased center and operator experience due to better knowledge of the LAA closure procedure and the device used.

The registry of Gloekler et al. [10] with 50 patients, who reported the first series of Amulet in their centers, also observed a relevant rate of serious procedure-related complications (12\%) with 3 cardiac tamponades, in 1 case leading to death, and 3 device embolizations.

The multicentre trial of 1,047 patients using the first generation ACP documented $4.97 \%$ major periprocedural events [5]. The ACP multicenter trial was based on retrospective data from 20 different centers including patients from first in December 2008. Data collection began years later and on 
operator voluntary contribution. The chance that a significant number of complications, possibly serious ones, could be missing and cannot be ruled out.

In the Amulet observational study with participation at highly experienced centers the rate of complications could be brought down to $2.7 \%$ [14].

The TEE follow-up of this study confirms excellent LAA sealing rate $(100 \%)$ with the Amplatzer Amulet. This is in line with all other studies.

Two device-related thrombi were detected. One thrombus disappeared after 6 weeks of treatment with $\mathrm{OAC}$, medication was then switched to aspirin alone. One 79-year-old patient refused TEE control and is still on lifelong treatment with OAC. In both patients there were no neurological problems. In the 79-year-old patient non-adherence to dual antiplatelet therapy was deemed possible due to moderate dementia (mini mental test 16 points). Implant result on angiogram showed full coverage of the LAA ostium (Fig. 2, 3A-C).

In the other patient the position of the device could have been a possible reason for thrombus formation.

The device had been implanted relatively deeply with the disc partially positioned in the neck of the LAA. Since the ostium of the LAA was not fully covered thrombus developed in the remaining pouch between the LAA ostium and the disc (Fig. 2, 2A-C).

A previous study of 169 consecutive patients evaluated the clinical outcome according to the position of the ACP disc [16]. There was no evidence of relevant differences in the incidence of devicerelated thrombi between patients with complete and incomplete disc coverage of the LAA ostium. However, study size and number of events were likely too small to detect significant differences.

At 12-months follow-up 7 patients had died unrelated to the device. Those patients were considerably older $(83.9 \pm 4.1$ years $)$ than the rest of the cohort ( $75 \pm 8.2$ years). Due to high $\mathrm{CHA}_{2} \mathrm{DS}_{2}-$ -VASc and HAS-BLED scores, elderly patients are likely to benefit from LAAO. However, future trials should examine the criteria on which to select older patients for LAAO.

Three patients were documented with ischemic stroke. TEE in these patients showed complete LAA sealing and could rule out device-related thrombus. Follow-up time was short and sample size not large enough to draw conclusions about the effectiveness of LAAO with the Amplatzer Amulet in reduction of thrombembolism.
Eight bleeding complications were reported. Two major bleedings, including 1 case of subarachnoid hemorrhage after a fall which occurred under dual antiplatelet therapy. Because of the design of the study, conclusions about bleeding risk compared between OAC and LAAO with the Amplatzer Amulet cannot be drawn. Still, keeping patients informed about increased bleeding risk under dual antiplatelet therapy after LAAO should be considered mandatory.

\section{Limitation of the study}

The retrospective and observational character of the study design does not exclude selection bias, although consecutive patients were considered. This single-center experience may not reflect clinical practice in other catheterization laboratories. Since this was a non-randomized design comparisons cannot be made to other devices or treatment with OAC. The 12-month follow-up is of insufficient length and the sample size was not large enough to draw definitive conclusions about the risk of thrombembolism and bleeding after LAAO with the Amplatzer Amulet.

\section{Conclusions}

Although minimizing procedure-related complications remains challenging, LAAO with the Amplatzer Amulet device showed high procedural success and excellent LAA sealing.

Conflict of interest: Honorarium provided by St. Jude Medical, Minneapolis, MN, USA to J.-W. Park.

\section{References}

1. Blackshear JL, Odell JA. Appendage obliteration to reduce stroke in cardiac surgical patients with atrial fibrillation. Ann Thorac Surg. 1996; 61(2): 755-759, doi: 10.1016/0003-4975(95)00887-X, indexed in Pubmed: 8572814.

2. Holmes DR, Reddy VY, Turi ZG, et al. PROTECT AF Investigators. Percutaneous closure of the left atrial appendage versus warfarin therapy for prevention of stroke in patients with atrial fibrillation: a randomised non-inferiority trial. Lancet. 2009; 374(9689): 534-542, doi: 10.1016/S0140-6736(09)61343-X, indexed in Pubmed: 19683639.

3. Holmes DR, Kar S, Price MJ, et al. Prospective randomized evaluation of the Watchman Left Atrial Appendage Closure device in patients with atrial fibrillation versus long-term warfarin therapy: the PREVAIL trial. J Am Coll Cardiol. 2014; 64(1): 1-12, doi: 10.1016/j.jacc.2014.04.029, indexed in Pubmed: 24998121.

4. Rodés-Cabau J, Champagne J, Bernier M. Transcatheter closure of the left atrial appendage: initial experience with the Amplatzer 
cardiac plug device. Catheter Cardiovasc Interv. 2010; 76(2): 186-192, doi: 10.1002/ccd.22499, indexed in Pubmed: 20665857.

5. Tzikas A, Shakir S, Gafoor S, et al. Left atrial appendage occlusion for stroke prevention in atrial fibrillation: multicentre experience with the AMPLATZER Cardiac Plug. EuroIntervention. 2016; 11(10): 1170-1179, doi: 10.4244/EIJY15M01_06, indexed in Pubmed: 25604089.

6. Freixa X, Chan JLK, Tzikas A, et al. The Amplatzer ${ }^{\mathrm{TM}}$ Cardiac Plug 2 for left atrial appendage occlusion: novel features and first-in-man experience. EuroIntervention. 2013; 8(9): 10941098, doi: 10.4244/EIJV8I9A167, indexed in Pubmed: 23339815.

7. Lam SC, Bertog S, Gafoor S, et al. Left atrial appendage closure using the Amulet device: an initial experience with the second generation amplatzer cardiac plug. Catheter Cardiovasc Interv. 2015; 85(2): 297-303, doi: 10.1002/ccd.25644, indexed in Pubmed: 25158644.

8. Cruz-Gonzáles I AD, Rama-Merchan JC, Pina-Gonzaález P, Sánchez PL, Serra A. Left Atrial Appendage Occlusion With the New AmuletTM Device: Feasibility, Safety and Short-term Efficacy. Rev Esp Cardiol. 2015; 68(8):719-30.

9. Freixa X, Abualsaud A, Chan J, et al. Left atrial appendage occlusion: initial experience with the Amplatzer ${ }^{\mathrm{TM}}$ Amulet $^{\mathrm{TM}}$. Int J Cardiol. 2014; 174(3): 492-496, doi: 10.1016/j.ijcard.2014.03.154, indexed in Pubmed: 24820753.

10. Gloekler S, Shakir S, Doblies J, et al. Early results of first versus second generation Amplatzer occluders for left atrial appendage closure in patients with atrial fibrillation. Clin Res Cardiol. 2015; 104(8): 656-665, doi: 10.1007/s00392-015-0828-1, indexed in Pubmed: 25736061.
11. Abualsaud A, Freixa X, Tzikas A, et al. Side-by-Side Comparison of LAA Occlusion Performance With the Amplatzer Cardiac Plug and Amplatzer Amulet. J Invasive Cardiol. 2016; 28(1): 34-38, doi: 10.1007/978-3-319-16280-5_12, indexed in Pubmed: 26716593.

12. Park JW, Bethencourt A, Sievert H, et al. Left atrial appendage closure with Amplatzer cardiac plug in atrial fibrillation: initial European experience. Catheter Cardiovasc Interv. 2011; 77(5): 700-706, doi: 10.1002/ccd.22764, indexed in Pubmed: 20824765.

13. Mehran R, Rao SV, Bhatt DL, et al. Standardized bleeding definitions for cardiovascular clinical trials: a consensus report from the Bleeding Academic Research Consortium. Circulation. 2011; 123(23): 2736-2747, doi: 10.1161/CIRCULATIONAHA.110.009449, indexed in Pubmed: 21670242.

14. Hildick-Smith D CJ, Diener H-C, Landmesser U, Paul V, Schmidt B, Settergren M, Teiger E, Tondo C. The Amulet observational study: a multicenter, prospective registry of the Amulet left atrial appendage closure device for stroke prevention in patients with atrial fibrillation. TCT 2016. [Presentation]. 2016.

15. Reddy VY, Holmes D, Doshi SK, et al. Safety of percutaneous left atrial appendage closure: results from the Watchman Left Atrial Appendage System for Embolic Protection in Patients with AF (PROTECT AF) clinical trial and the Continued Access Registry. Circulation. 2011; 123(4): 417-424, doi: 10.1161/CIRCULATIONAHA.110.976449, indexed in Pubmed: 21242484.

16. Wolfrum M, Attinger-Toller A, Shakir S, et al. Percutaneous left atrial appendage occlusion: Effect of device positioning on outcome. Catheter Cardiovasc Interv. 2016; 88(4): 656-664, doi: 10.1002/ccd.26646, indexed in Pubmed: 27465268. 